\title{
Immune System-Based Optimization for Power Economic Dispatch Problem
}

\author{
Chao-Lung Chiang
}

\begin{abstract}
This paper proposes a hybrid algorithm of an immune system-based optimization with multiplier update method (ISBO-MU) for the power economic dispatch problem (PEDP) of generating units with valve-point effects. The immune system-based optimization (ISBO) with a migrating function can actively search and effectively explore solutions. Multiplier update (MU) was employed to avoid the Lagrange function which deforms searching difficult. The investigated approach synthesizes ISBO and MU, and its merits are that it can automatically regulate the randomly given penalty to a suitable value and requiring only a small-size population for the PEDP of considering generating plants having valve-point effects. Simulated results of a 13-unit practical system dividing into two cases demonstrate that the proposed ISBO-MU is more suitable than the former studies in the actual economic dispatch application of power system.
\end{abstract}

Index Terms-power economic dispatch problem, immune system-based optimization, valve-point effects.

\section{INTRODUCTION}

The input-output characteristics of power generating plant are highly nonlinear and contain discontinuities due to the influences of valve-point loading. When each steam admission valve in a turbine starts to open, wire drawing effects will occur giving rise to a sharp increase in losses [1]. Actually, in the PEDP, the cost function for each generating unit has been approximately represented by a single quadratic function, and the valve-point effects [2] were ignored. This would often introduces inaccurate scheduling. However, since the cost curve of a power unit is highly nonlinear, containing discontinuities owing to valve-point effects, the cost function is more realistically denoted as a segmented piecewise nonlinear function [3] rather than a single quadratic function. The PEDP considering of power generators with valve-point effects is represented as a nonsmooth optimization problem having complex and non-convex characteristics with heavy equality and inequality constraints, which makes the challenge of finding the global optimum hard. Methods, which avoid the actual unit curve model without sacrificing calculation time will prove very valuable. Some studies of the PEDP, such as a modified artificial bee colony algorithm, (MABC)[4], a hybrid particle swarm optimization and gravitational search algorithm (PSOGSA) based on fuzzy logic (FL), called modified hybrid FL-PSO-GSA (FPSOGSA)[5], a shuffled differential evolution (SDE)[6], a modified shuffled frog leaping algorithm (MSFLA)[7], a collective neuro-dynamic optimization (CNO)[8], a fuzzy based hybrid particle swarm

Chao-Lung Chiang, Department of Electronic Engineering, Nan Kai University of Technology, Nan-Tou 542, Taiwan, ROC. optimization-differential evolution (FBHPSO-DE) [9], an integrated mixed-integer linear programming (MILP) and the interior point method (IPM), called as MILP-IPM[10], a hybrid method named BBOSB by combining biogeography-based optimization (BBO) with brain storm optimization (BSO)[11], a hybrid method based on modified particle swarm optimization and genetic algorithm (MPSO-GA)[12], an originated from harmony search algorithm (HSA) called chaotic improved harmony search algorithm (CIHSA)[13], a dimensional steepest decline Method (DSD) [14], a hybridization of the harmony search (HHS) [15], a $\Theta$-particle swarm optimization ( $\Theta$-PSO) [16], a Firefly algorithm (FA) [17], a new fuzzy adaptive hybrid PSO (FAPSO) [18], an artificial Bee Colony (ABC) [19], an estimation of distribution and differential evolution (ED-DE) [20], a self-adaptive real coded genetic algorithm using Taguchi method (TSARGA) [21], a combining the DE with the generator of chaos sequences and sequential quadratic programming (SQP) technique (DEC-SQP) [22], and a hybrid multi-agent based PSO (HMAPSO) [23], have considered the valve-pint effects for PEDPs.

ISBO are observed in nature immunology, immune function and the principle of inspiration. ISBO is a very complex biological system, it illustrates the organism's ability to fight harmful foreign entities. Now it has attracted the interest of many researchers and has been successfully used in various research fields [24 27].

\section{SYSTEM FORMULATION}

The PEDP can be described as an optimization (minimization) process with objective [1]:

$$
\text { Minimize } \quad \sum_{i=1}^{n_{p}} F_{i}\left(P_{i}\right)
$$

Where $F_{i}\left(P_{i}\right)$ is the fuel cost function of the $i^{\text {th }}$ unit, $P_{i}$ is the power generated by the $i^{\text {th }}$ generator, and $n_{P}$ is the number of dispatchable units. Considering the actual cost curve for each power unit, the valve-point effects must be involved in the cost model. Therefore, the sinusoidal function is incorporated into the quadratic function [3]. The cost function for solving the valve-point effects of generating units is accurately represented as [28]:

$$
F_{i}\left(P_{i}\right)=a_{i}+b_{i} P_{i}+c_{i} P_{i}^{2}+\left|e_{i} \times \sin \left(f_{i} \times\left(P_{i}^{\min }-P_{i}\right)\right)\right|
$$

Where $a_{i}, b_{i}$, and $c_{i}$ are the fuel cost coefficients of the $i^{\text {th }}$ unit, and $e_{i}$ and $f_{i}$ are fuel cost coefficients of the $i^{\text {th }}$ unit with valve-points loadings. Subject to the equality constraint of 
the power balance as:

$$
\sum_{i=1}^{n_{p}} P_{i}=P_{d}+P_{L}
$$

Where $P_{d}$ is the system load demand and $P_{L}$ is the transmission loss, and generating capacity constraints as:

$$
P_{i}^{\min } \leq P_{i} \leq P_{i}^{\max }, \quad i=1, \cdots, n_{p}
$$

Where $P_{i}^{\min }$ and $P_{i}^{\max }$ are the minimum and maximum power outputs of the $i^{\text {th }}$ unit.

\section{The PROPOSED AlgORITHM (ISBO-MU)}

\section{A. The Immune System-Based Optimization (ISBO)}

In ISBO [15], mimicking the clone generation, proliferation and maturation of these biological principles. Based on the clonal selection principle of ISBO the main step is the activation of the antibody encounters an antigen the cells proliferation and differentiation, by carrying out affinity maturation process and maturity, eliminating old antibodies to maintain the antibodies diversity and avoid premature convergence, selection with antigen affinity more of those antibodies. In order to optimize the simulation of the ISBO, the antibodies and affinity, respectively, as the feasible solutions and the objective function.

\section{B. The Multiplier Updating (MU)}

Considering the nonlinear programming problem with general constraints as follows:

$$
\begin{array}{lll} 
& \min _{x} f(x) & \\
\text { subject to } \quad & h_{k}(x)=0, \quad k=1, \ldots, m_{e} \\
& g_{k}(x) \leq 0, \quad k=1, \ldots, m_{i}
\end{array}
$$

Where $x$ represents a $n_{C}$-dimensional variable, and the $h_{k}(x)$ and $g_{k}(x)$ stand for equality and inequality constraints, respectively.

Penalty function method is usually used to manage the evolutionary algorithm in the constraint. Such technology through the penalty constraint violation of the original constraint problem is converted to unconstrained problem. The penalty function method the concept and implementation is simple. However, its main limitation is that each constraint be punished to the extent. These penalty terms have a certain weakness, when the penalty parameter is large, these weaknesses will become fatal. Such a penalty function tends to generally located in the best point of the feasible region near the border of discomfort. The Lagrangian method can obviously overcome the penalty study drawbacks. The augmented Lagrange function $(A L F)$ [29] for constrained optimization problems is defined as:

$$
L_{a}(x, v, v)=f(x)+\sum_{k=1}^{m_{e}} \alpha_{k}\left\{\left[h_{k}(x)+v_{k}\right]^{2}-v_{k}^{2}\right\}+\sum_{k=1}^{m_{i}} \beta_{k}\left\{\left\langle g_{k}(x)+v_{k}\right\rangle_{+}^{2}-v_{k}^{2}\right\}
$$

Where $\alpha_{k}$ and $\beta_{k}$ are the positive penalty parameters, and the corresponding Lagrange multipliers $v=\left(v_{1}, \ldots, v_{m_{e}}\right)$ and $v=\left(v_{1}, \ldots, v_{m_{i}}\right) \geq 0$ are associated with equality and inequality constraints, respectively.

\section{The Proposed ISBO-MU}

The contour of $A L F$ does not change the shape between generations while system constrains are linear. Consequently, the contour of $A L F$ is just a shift or prejudice, in relation to the original target function, $f(x)$. Consequently, small penalty parameters can be used in the MU. However, the shape of contour of $\mathrm{La}$ is changed by penalty parameters while the constraints are nonlinear, demonstrating that large penalty parameters still create computational difficulties. Adaptive penalty parameters of the MU are employed to alleviate the above difficulties. Table 1 shows computational procedures of the MU. The original objective function can be scaled to avoid the ill conditioning by updating penalty parameters and multipliers.

Table 1: COMPUTATIONAL PROCEDURES OF The PRoPosed ISBO-MU

Step 1. Set the initial iteration $l=0$. Set initial multiplier, $v_{k}^{l}=v_{k}^{0}=0, k=1, \ldots, m_{e}, \quad v_{k}^{l}=v_{k}^{0}=0, k=1, \ldots, m_{i}$, and the initial penalty parameters, $\alpha_{k}>0, k=1, \ldots, m_{e}$ and $\beta_{k}>0$, $k=1, \ldots, m_{i}$. Set tolerance of the maximum constraint violation, $\varepsilon_{k}$ (e.g. $\varepsilon_{k}=10^{32}$ ), and the scalar factors, $\omega$ ${ }_{1}>1$ and $\omega_{2}>1$.

Step 2. Use a minimization solver, e.g. ISBO, to solve $L_{a}\left(x, v^{l}, v^{l}\right)$. Let $x_{b}^{l}$, be a minimum solution to the problem $L_{a}\left(x, v^{l}, v^{l}\right)$.

Step 3. Evaluate the maximum constraint violation as $\hat{\varepsilon}_{k}=\max \left\{\max _{k}\left|h_{k}\right|, \max _{k}\left|\max \left(g_{k},-v_{k}\right)\right|\right\}$, and establish the following sets of equality and inequality constraints whose violations have not been improved by the factor $\omega_{l}$ :

$$
\begin{aligned}
& I_{E}=\left\{k:\left|h_{k}\right|>\frac{\varepsilon_{k}}{\omega_{1}}, \quad k=1, \cdots, m_{e}\right\} \\
& I_{I}=\left\{k:\left|\max \left(g_{k}, \quad-v_{k}\right)\right|>\frac{\varepsilon_{k}}{\omega_{1}}, \quad k=1, \cdots, m_{i}\right\}
\end{aligned}
$$

Step 4. If $\hat{\varepsilon}_{k} \geq \varepsilon_{k}$, let $\alpha_{k}=\omega_{2} \alpha_{k}$ and $v_{k}^{l+1}=v_{k}^{l} / \omega_{2}$ for all $k \in I_{E}$, let $\beta_{k}=\omega_{2} \beta_{k}$ and $v_{k}^{l+1}=v_{k}^{l} / \omega_{2}$ for all $k \in I_{I}$, and go to step7. Otherwise, go to step 5.

Step 5. Update the multipliers as follows:

$$
\begin{gathered}
v_{k}^{l+1}=h_{k}\left(x_{b}^{l}\right)+v_{k}^{l} \\
v_{k}^{l+1}=\left\langle g_{k}\left(x_{b}^{l}\right)+v_{k}^{l}\right\rangle_{+}=v_{k}^{l}+\max \left\{g_{k}\left(x_{b}^{l}\right),-v_{k}^{l}\right\}
\end{gathered}
$$

Step 6. If $\hat{\varepsilon}_{k} \leq \varepsilon_{k} / \omega_{1}$, let $\varepsilon_{k}=\hat{\varepsilon}_{k}$ and go to step 7. Otherwise, let $\alpha_{k}=\omega_{2} \alpha_{k}$ and $v_{k}^{l+1}=v_{k}^{l} / \omega_{2}$ for all $k \in I_{E}$, and let $\beta_{k}=\omega_{2} \beta_{k}$ and $v_{k}^{l+1}=v_{k}^{l} / \omega_{2}$ for all $k \in I_{I}$. Let $\varepsilon_{k}=\hat{\varepsilon}_{k}$ and go to step 7 .

Step 7. If the maximum iteration reaches, stop. Otherwise, repeat steps 2 to 6 .

In Table 1, steps 3, 4 and 6 are adopted to reform the constraint violation and update penalty parameters. Step 3 
evaluates the maximum constraint violation $\hat{\varepsilon_{k}}$, which is the maximum value between the maximum equality constraint violation $\left(\left|h_{k}\right|, k=1, \ldots, m_{e}\right)$ and the maximum inequality constraint violation $\left(\left|\max \left(g_{k},-v_{k}\right)\right|, k=1, \ldots, m_{i}\right)$. In step 4 , if constraint violations have not been improved, such that $\hat{\varepsilon_{k}} \geq \varepsilon_{k}$, the penalty parameters are increased by a factor $\omega_{2}$ (e.g. $\omega_{2}=10$, in this paper) and the multipliers are reduced by the same factor, maintaining the product of penalty parameters time multipliers unchanged. Step 6 uses factor $\omega_{1}$ (e.g. $\omega_{1}=4$, in this paper) to check whether this factor reduces the level of constraint violation. The penalty parameters and multipliers are updated in this step if $\omega_{1}$ does not reduce the constraint violation.

The flow chart of the presented method is displayed below in figure 1, and more details of the MU have shown in [30] and [31].

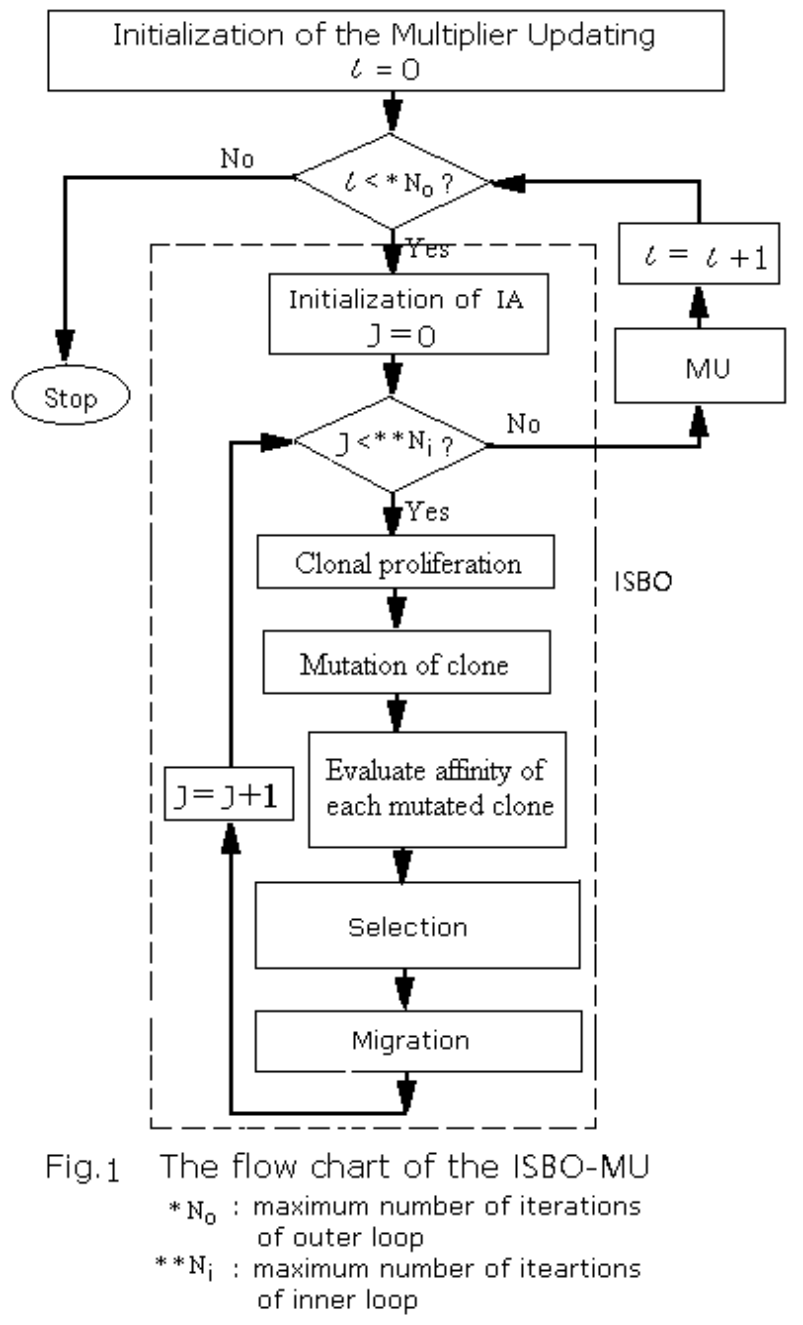

\section{SYSTEM SIMULATIONS}

Use of a practical power system to illustrate the proposed ISBO-MU in obtaining the solution of the effectiveness of the quality aspect. In this example, the MU algorithm was used for managing the system constraints of this example. The ISBO-MU was directly coded using real values, and were implemented on a personal computer $(\mathrm{CPU} 3.0 \mathrm{GHz})$ in FORTRAN-90 software language. Setting factors used in this example were as follows: the population size $n_{p}$ was respectively set to 5 . The iteration numbers of the outer loop and inner loop were set to (outer, inner) as $(10,500)$. This test system contained thirteen dispatching units addressing power generator with valve-point effects for a load demand $\left(P_{d}\right)$ of $1800 \mathrm{MW}$ and with/without the transmission loss $\left(P_{L}\right)$. The system data of this example originated from [28]. Executing this example can be illustrated as follows:

$$
\begin{aligned}
& L_{a}(x, v, v)=f(x)+\alpha_{1}\left\{\left[h_{1}(x)+v_{1}\right]^{2}-v_{1}^{2}\right\} \\
& \text { objective: } \min _{x=\left(P_{1}, P_{2}, \cdots P_{13}\right)} f(x)=\sum_{i=1}^{13} F_{i}\left(P_{i}\right) \\
& h_{1}: \quad \sum_{i=1}^{13} P_{i}-P_{d}-P_{L}=0 \\
& g_{1}: \quad P_{1}-P_{1}^{\max } \leq 0 \\
& \text { subject to } g_{13}: \quad P_{13}-P_{13}^{\max } \leq 0 \\
& g_{14}: \quad P_{1}^{\min }-P_{1} \leq 0 \\
& \vdots \\
& g_{26}: \quad P_{13}^{\min }-P_{13} \leq 0
\end{aligned}
$$

The implementations of the proposed algorithm for this example has one objective function with ten variable parameters, $\left(P_{1}, P_{2}, \ldots, P_{13}\right)$, one equality constraint, $\left(h_{1}\right)$, and twenty-six inequality constraints, $\left(g_{1}\right.$ to $\left.g_{26}\right)$. In order to show that the proposed ISBO-MU can obtain the best solution that fully meets the requirements of the system constraints. An evaluation of the total number of system constraint violations $(S C V)$ described in (10).

$$
S C V=\left|h_{1}\right|+\sum_{k=1}^{26} \max \left\{g_{k}, \quad 0.0\right\}
$$

\section{A. Case 1: With considering the transmission loss}

The purpose of case 1 is to demonstrate that the proposed ISBO-MU for the power system of the actual PEDP effectiveness of considering the transmission loss. This case will suggest the ISBO-MU with MABC [4], the FPSOGSA [5], SDE [6] and MSFLA [7] for comparison. The comparative results are shown in Table 2 , where $T P$ and $T C$ respectively represent the total power and the total cost. Studies of the MABC [4], FPSOGSA [5], SDE [6], and MSFLA [7], because the $S C V$ is not zero, this means that the first four methods can not completely meet the system constraints. Therefore, the previous algorithms' results are not feasible solutions. Obtained results of the proposed ISBO-MU method not only provide sufficient load demand and transmission loss, but the results obtained from the proposed algorithm have lower costs than that obtained by the earlier researches. 
Table 2: Compared Results of best Cost For Previous Methods and The Proposed ISBO-MU (Load 1800MW with transmission

\begin{tabular}{|c|c|c|c|c|c|}
\hline Methods & МАВС [4] & FPSOGSA[5] & SDE[6] & MSFLA[7] & ISBO-MU \\
\hline $\mathrm{P}(\mathrm{G} 1)$ & 448.7989505145 & 448.7990 & 448.80 & 540.5290 & 448.79399 \\
\hline $\mathrm{P}(\mathrm{G} 2)$ & 299.1993059732 & 297.9312 & 297.93 & 225.0747 & 299.18624 \\
\hline $\mathrm{P}(\mathrm{G} 3)$ & 224.3994800693 & 223.3374 & 223.30 & 207.9648 & 224.39848 \\
\hline $\mathrm{P}(\mathrm{G} 4)$ & 109.8665500881 & 109.8666 & 109.85 & 69.0974 & 109.86640 \\
\hline $\mathrm{P}(\mathrm{G} 5)$ & 109.8665501037 & 109.8666 & 109.85 & 84.9624 & 109.86746 \\
\hline $\mathrm{P}(\mathrm{G} 6)$ & 109.8665502275 & 159.7331 & 159.71 & 94.7620 & 109.74770 \\
\hline $\mathrm{P}(\mathrm{G} 7)$ & 109.8665500941 & 109.8666 & 109.86 & 106.9725 & 109.86534 \\
\hline $\mathrm{P}(\mathrm{G} 8)$ & 109.8665500558 & 60.0000 & 60.00 & 109.0098 & 109.86322 \\
\hline $\mathrm{P}(\mathrm{G} 9)$ & 109.8665500980 & 109.8666 & 109.82 & 108.3280 & 109.86641 \\
\hline $\mathrm{P}(\mathrm{G} 10)$ & 40.0000000000 & 40.0000 & 40.00 & 79.6389 & 40.00120 \\
\hline $\mathrm{P}(\mathrm{G} 11)$ & 40.5518593925 & 40.0000 & 40.00 & 63.7670 & 40.00120 \\
\hline $\mathrm{P}(\mathrm{G} 12)$ & 55.0000000013 & 55.0000 & 55.00 & 58.0643 & 55.00108 \\
\hline $\mathrm{P}(\mathrm{G} 13)$ & 55.0000000000 & 55.0000 & 55.00 & 72.9603 & 55.00112 \\
\hline$T P(\mathrm{MW} / \mathrm{h})$ Reported & 1822.148896617 & 1819.13 & 1819.13 & 1821.0903 & 1821.45984 \\
\hline$P_{L}(\mathrm{MW} / \mathrm{h})$ Reported & 22.1488966179 & 19.13 & 19.13 & 21.0903 & 21.45983 \\
\hline$T C(\$ / \mathrm{h})$ Reported & 18127.782085 & 18134.49 & 18134.49 & 17944.84 & 18118.5052 \\
\hline Actual $\Sigma \mathrm{P}(\mathrm{MW} / \mathrm{h})$ & 1822.148896618 & 1819.2671 & 1819.1200 & 1821.1311 & 1821.45984 \\
\hline Actual $P_{L}(\mathrm{MW} / \mathrm{h})$ & 21.454273 & 18.4344 & 18.4305 & 21.1313 & 21.45983 \\
\hline Actual TC $(\$ / h)$ & 18127.782085 & 18134.3946 & 18134.5130 & 18954.2750 & 18118.5052 \\
\hline$S C V$ & 0.6946 & 0.8327 & 0.6895 & 0.0002 & 0.0000 \\
\hline CPU Time (Sec) & - & - & - & 52.33 & 24.63 \\
\hline
\end{tabular}

Table 3: Comparison of Previous Methods and The Proposed ISBO-MU (Load 1800MW without transmission loss)

\begin{tabular}{c|cccc}
\hline Items & Min. Cost $\mathbf{\$} / \mathbf{h})$ & Mean Cost $(\mathbf{\$} / \mathbf{h})$ & Max. Cost $\mathbf{\$} / \mathbf{h})$ & SD (\$/h) \\
Methods & & & & \\
\hline ISBO-MU & $\mathbf{1 7 9 6 3 . 8 2 9 2 0}$ & $\mathbf{1 7 9 6 3 . 8 7 6 7 8}$ & $\mathbf{1 7 9 6 3 . 9 6 6 5 8}$ & $\mathbf{0 . 0 2 7 9 6 4}$ \\
MABC [4] & 17963.82920 & 17963.82933 & 17963.83045 & 0.000226 \\
DSD [14] & 17963.8292 & 18154.562 & 18358.310 & - \\
HHS [15] & 17963.8293 & 17972.4822 & - & 2.4185 \\
@-PSO [16] & 17963.8297 & 17965.2055 & 17980.2030 & 4.3807 \\
FA [17] & 17963.83 & 18029.16 & 18168.80 & 148.542 \\
SDE [6] & 17963.83 & - & - & - \\
FAPSO [18] & 17963.84 & 17969.9187 & 17976.35 & - \\
ABC [19] & 17963.86 & 17987.22 & 17995.11 & - \\
ED-DE [20] & 17963.86 & 17972.70 & 17975.89 & - \\
TSARGA [21] & 17963.94 & 17974.31 & 18264.23 & 3.18 \\
DEC-SQP [22] & 17963.9401 & 17973.1339 & 17984.8105 & 1.9735 \\
HMAPSO [23] & 17969.31 & 17969.31 & 17969.31 & 0.00 \\
\hline
\end{tabular}




\section{B. Case 2: Without considering the transmission loss}

The same 13-unit power system as case1 was used in case 2 , except that this case didn't consider the transmission loss. More comparative results are also listed in Table 3, where $\mathrm{SD}$ is a standard deviation of 50 trials. The results Obtained by the proposed ISBO-MU are also compared with twelve pervious algorithms. It is evident from Table 3 that the proposed approach performed better than ten previous methods of HHS [15], $\Theta$-PSO [16], FA [17], SDE [6], FAPSO [18], ABC [19], ED-DE [20], TSARGA [21], DEC-SQP [22], and HMAPSO [23] in best total cost (Min. Cost). The proposed ISBO and two early methods of MABC [4] and DSD [14], all three have effective searching performance. At the same time, performance in standard deviation of the proposed ISBO-MU is also less than most literature results.

Convergence curve of the total cost for this practical 13 -unit system and the total cost values obtained from the proposed algorithm for best 50 trials have been given in figures of 2 and 3 , respectively.

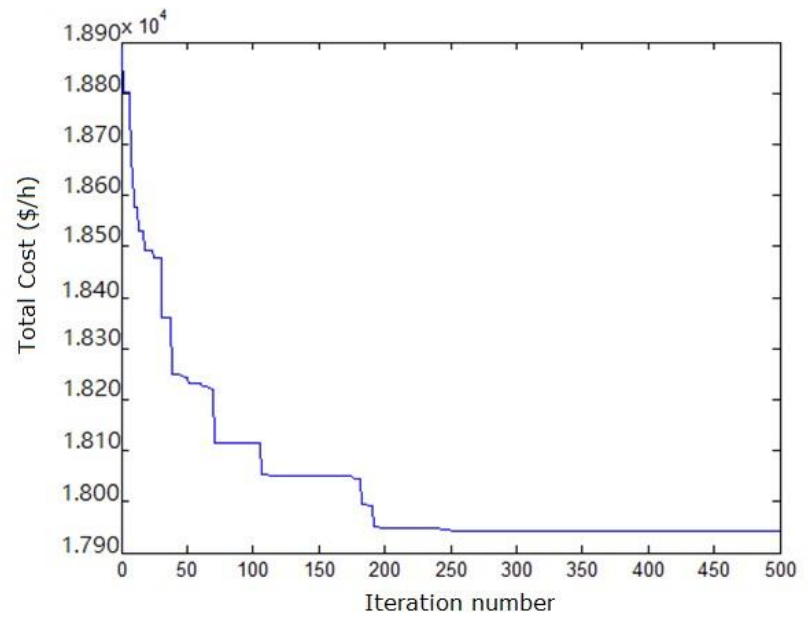

Fig. 2 Convergence curve of the best total cost values obtained from the proposed ISBO-MU

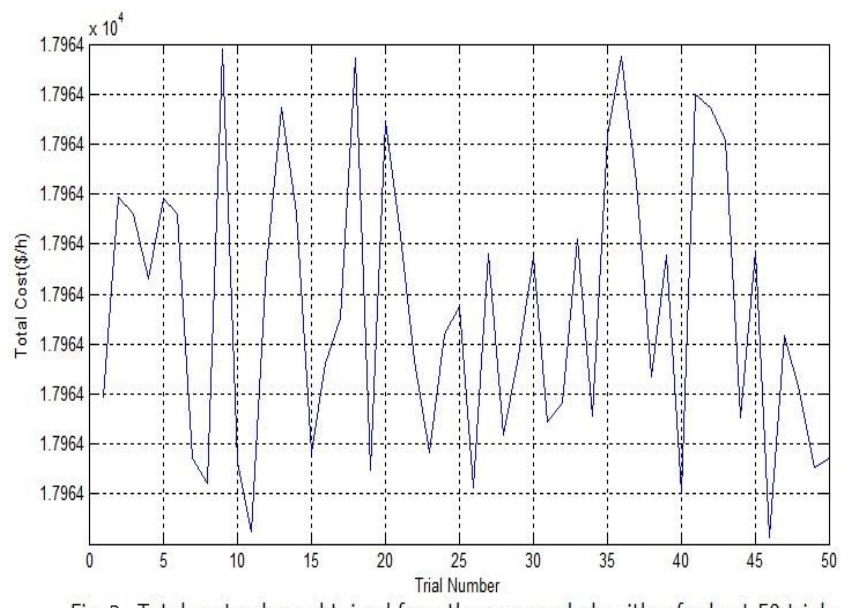

Fig. 3 Total cost values obtained from the proposed algorithm for best 50 trials

As a result, two cases have clearly shown that due to the proposed study is more suitable than previous researches in application because the proposed ISBO-MU is easy to implement, and has a satisfactory result of solving the PEDP having power generators having valve-point effects.

\section{V.CONCLUSION}

Actual PEDP is complicated because the valve-point effects must be considered. ISBO helps the proposed algorithm search and explore efficiently. The MU assists the proposed method to avoid $A L F$ deformation and makes solving the search difficult. The proposed study combines ISBO and MU together, giving it the advantage of automatically adjusting a randomly given penalty to an appropriate value and requiring only a small population. System simulated results of two cases show that the proposed study has more benfits for solving the PEDP with valve-point effects than the previous researches for power systems.

\section{ACKNOWLEDGMENT}

Financial support given to this research by the Ministry of Science and Technology, Taiwan, R.O.C. under Grant No. MOST 108-2637-E-252-005 is greatly appreciated.

\section{REFERENCES}

[1] A. J. Wood and B. F. Wollenberg, Power generation, operation, and control, New York: Wiley \& Sons, the $2^{\text {nd }}$ ed., 1996.

[2] C. E. Lin, G. L. Viviani, "Hierarchical economic dispatch for piecewise quadratic cost functions," IEEE Trans. PAS, vol. 103, no. 6, 1984, pp. 1170-1175.

[3] D. C. Walters and G. B. Sheble, "Genetic algorithm solution of economic dispatch with valve point loading," IEEE Trans. Power Syst., vol. 8, no. 3, 1993, pp. 1325-1332.

[4] D. C. Secui, "A new modified artificial bee colony algorithm for the economic dispatch problem," Energy Conversion and Management, vol. 89, 2015, pp. 43-62.

[5] S. Duman, N. Yorukeren, and I. H. Altas, "A novel modified hybrid PSOGSA based on fuzzy logic for non-convex economic dispatch problem with valve-point effect," Electrical Power and Energy Systems, vol. 64, 2015, pp.121-135.

[6] A. S. Reddy and K. Vaisakh, "Shuffled differential evolution for large scale economic dispatch," Electrical Power System Research, vol. 96, 2013, pp. 237-245.

[7] P. Roy, P. Roy, and A. Chakrabarti, "Modified shuffled frog leaping algorithm with genetic algorithm crossover for solving economic load dispatch problem with valve-point effect," Applied Soft Computing, vol. 13, 2013, pp. 4244-4252.

[8] T. Wang, X. He, T. Huang, C. Li, and W. Zhang, "Collective neuro dynamic optimization for economic emission dispatch problem considering valve point effect in microgrid," Neural Networks, vol. 93, 2017, pp. 126-136.

[9] E. Naderia, A. Azizivahed, H. Narimani, M. Fathi, and M. R. Narimani, "A comprehensive study of practical economic dispatch problems by a new hybrid evolutionary algorithm," Applied Soft Computing, vol. 61, 2017, pp.1186-1206.

[10] S. Pan, J. Jian, and L. Yang, "A hybrid MILP and IPM approach for dynamic economic dispatch with valve-point effects," Electrical Power and Energy Systems, vol. 97, 2018, pp. 290-298.

[11] G. Xiong and D. Shi, "Hybrid biogeography-based optimization with brain storm optimization for non-convex dynamic economic dispatch with valve-point effects," Energy, vol. 15, 2018, pp. 424-435.

[12] H. Barati and M. Sadeghi, "An efficient hybrid MPSO-GA algorithm for solving non-smooth/non-convex economic dispatch problem with practical constraints," Ain Shams Engineering Journal, vol. 9, 2018, pp. 1279-1287.

[13] H. Rezaie, M. H. Kazemi-Rahbar, B. Vahidi, and H. Rastegar, "Solution of combined economic and emission dispatch problem using a novel chaotic improved harmony search algorithm," Journal of Computational Design and Engineering, vol. 6, no. 3, 2019, pp. 447-467. 
[14] Junpeng Zhan, Q. H. Wu, Chuangxin Guo, and Xiaoxin Zhou, "Economic Dispatch With Non-Smooth Objectives-Part II: Dimensional Steepest Decline Method," IEEE Transactions on power systems, vol. 30, no. 2, MARCH 2015, pp. 722-733.

[15] V. R. Pandi, B. K. Panigrahi, R. C. Bansal, S. Das, and A. Mohapatra, "Economic Load Dispatch Using Hybrid Swarm Intelligence Based Harmony Search Algorithm," Electric Power Components and Systems, vol. 39, 2011, pp. 751-767.

[16] V. Hosseinnezhad and E. Babaei,"Economic load dispatch using $\Theta-P S O, "$ Electrical Power and Energy Systems, vol. 49, 2013, pp. 160-169.

[17] X. -S. Yang, S. S. Sadat Hosseini, and A. H. Gandomi, "Firefly algorithm for solving non-convex economic dispatch problems with valve loading effect," Appl. Soft Comput., vol. 12, no. 3, 2012, pp. $1180-1186$.

[18] T. Niknam, "A new fuzzy adaptive hybrid particle swarm optimization algorithm for non-linear, non-smooth and non-convex economic dispatch problem," Applied Energy, vol. 87, 2010, pp. $327-339$.

[19] S. Hemamalinia and S. P. Simon, "Artificial Bee Colony Algorithm for Economic Load Dispatch Problem with Non-smooth Cost Functions," Electric Power Components and Systems, vol. 38, 2010, pp.786-803.

[20] Y. Wang, B. Li, and T. Weise, "Estimation of distribution and differential evolution cooperation for large scale economic load dispatch optimization of power systems," Inf. Sci., vol. 180, no. 12, 2010, pp. 2405-2420.

[21] Subbaraj P, Rengaraj R, Salivahanan S, "Enhancement of self-adaptive real coded genetic algorithm using Taguchi method for economic dispatch problem," Applied Soft Comput., vol. 11, pp83-92, 2011.

[22] Leandro dos Santos Coelho and Viviana Cocco Mariani, "Combining of chaotic differential evolution and quadratic programming for economic dispatch optimization with valve-point effect," IEEE Transactions on power systems, vol. 21, no. 2,MAY 2006, pp. 989-996.

[23] R. Kumar, D. Sharma, A. Sadu, "A hybrid multi-agent based particle swarm optimization algorithm for economic power dispatch," Energy Systems, vol. 33, no. 1, 2011, pp. 115-123.

[24] S. Hemamalini and S. P. Simon, "Dynamic economic dispatch using artificial immune system for units with valve-point effect," Electrical Power and Energy Systems, vol. 33, 2011, pp. 868-874.

[25] M. Basu, "Artificial immune system for combined heat and power economic dispatch," Electrical Power and Energy Systems, vol. 43, 2012, pp. 1-5.

[26] X. Kong, D. Liu, J. Xiao, and C. Wang, "A multi-agent optimal bidding strategy in microgrids based on artificial immune system," Energy, vol. 189, Article 116154, 2019.

[27] D. Corus, P. S. Oliveto, and D. Yazdani, "Artificial immune systems can find arbitrarily good approximations for the NP-hard number partitioning problem," Artificial Intelligence, vol. 274, 2019, pp. 180-196.

[28] N. Sinha, R. Chakrabarti, and P. K. Chattopadhyay, "Evolutionary programming techniques for economic load dispatch," IEEE Evol. Comput., vol. 7, no. 1, 2003, pp. 83-94.

[29] Z. Michalewicz and M. Schoenauer, "Evolutionary algorithms for constrained parameter optimization problems," Evolutionary Computation, vol. 4, no. 1, 1996, pp. 1-32.

[30] C. L. Chiang, C. T. Su, and F. S. Wang, "Augmented Lagrangian method for evolutionary optimization of mixed-integer nonlinear constrained problems," Intern. Math. J., vol. 2, no. 2, 2002, pp. 119-154.

[31] C. L. Chiang, "Improved genetic algorithm for power economic dispatch of units with valve-point effects and multiple fuels," IEEE Transactions on Power Systems, vol. 20, no. 4, 2005, pp. 1690-1699. 